\title{
Clinical Research Support Shared Resource
}

National Cancer Institute

\section{Source}

National Cancer Institute. Clinical Research Support Shared Resource. NCI Thesaurus.

Code C39360.

The Clinical Research Shared Resource provides Cancer Center investigators with assistance in identification of prospective patients and determination of patient interest and eligibility to participate in clinical trials, in procurement and maintenance of the informed consent process, coordination of follow-up visits in conformance with protocol, hospital admission and discharge planning, coordination and safe administration of investigational agents, toxicity monitoring and reporting in compliance within Cancer Center and FDA regulations and the guidelines of GCP. 